\title{
MECHANISMS OF EVOLUTION IN ANIMAL MITOCHONDRIAL DNA*
}

\author{
Wesley M. Brown \\ Division of Biological Sciences \\ University of Michigan \\ Ann Arbor, Michigan 48109
}

The mitochondrial genome of multicellular animals is a covalently closedcircular duplex DNA that is, in many respects, ideally suited for evolutionary studies. It is relatively easy to prepare in highly purified form and in amounts sufficiently large to allow its analysis by a number of different methods. It is small and simple enough to be easily characterizable by these methods. It appears to lack many of the complicating features-e.g., large size, intervening sequences, and the presence of different repetition classes-that are found in nuclear DNA, chloroplast DNA, and the mitochondrial DNA of lower eukaryotes and plants. Also, it is possible to correlate the changes observed in animal mitochondrial DNA with time, because the evolutionary history of the animals themselves is known from the fossil record.

There is evidence that, at least in vertebrates, the mitochondrial gene content and relative gene order is very similar, if not identical, among different species. $^{1-7}$ Despite this apparently conservative feature, the sequence of animal mitochondrial DNA (mtDNA) appears to evolve very rapidly, at least among

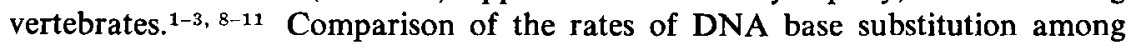
mammalian species indicates that mtDNA evolves 5 to 10 times faster than single-copy nuclear DNA. ${ }^{3}$

Three central questions regarding the evolution of animal mtDNA are: What kind of evolutionary change does animal mtDNA undergo? What mechanisms are responsible for the generation of change? How can the high rate of change observed be tolerated in the genome of an intracellular organelle whose function is so vital for the cell? Fairly substantial answers to the first two questions can be provided. At present, only a speculative answer to the third question is possible, although a more substantial answer may be available in a few years when more is known about the exact functions carried out by the mtDNA gene products.

Although this report contains previously unpublished data that bear on the above questions, I have also relied on past studies (my own and those of others) to provide support for the conclusions presented. In this respect I have tried to select papers that are representative of the studies that bear on a given point, and have made no attempt to provide a comprehensive survey of the literature. As a consequence, many contributions to this area of investigation have not been cited.

* Supported by the National Science Foundation (Grants DEB78-02841 and DEB76-20599). 


\section{Materials AND Methods}

\section{Sources of $m t D N A$}

The tissues and cell lines used for mtDNA preparation for many of the species examined have been described previously.3.11-14 Xenopus laevis and $\boldsymbol{X}$. borealis mtDNAs were gifts from Richard Hallberg. Primary cultures of chimpanzee and Talapoin monkey cells were obtained from Adeline Hackett (Naval Biomedical Research Laboratory, Oakland, Calif.), Bush Baby liver was obtained from John Allman (Biology Division, California Institute of Technology, Pasadena, Calif.). The remaining sources were tissues (usually liver) that were taken from animals obtained commercially or collected in their native habitats. A complete listing of the species examined and the sources of mtDNA is given in TABLE 1 (see REsults).

\section{Preparation, Labeling and Strand Separation of $m t D N A$}

The preparation of mtDNA from cells and tissues was as described..$^{3,11-11}$ For base compositional analysis. mtDNA was prepared from cultured cells grown for $96 \mathrm{hr}$ in medium containing $5 \times 10^{-4} \mathrm{M}$ sodium phosphate, $\mathrm{pH} 7.4$ and $5 \mu \mathrm{Ci} / \mathrm{ml}$ of carrier-free $: 2 \mathrm{P}$ to ensure uniform labeling of all bases. The $\mathrm{CsCl}$, propidium diiodide gradient profiles obtained during mtDNA preparation are shown in Figure 1. The purity of the mtDNA obtained is indicated by the

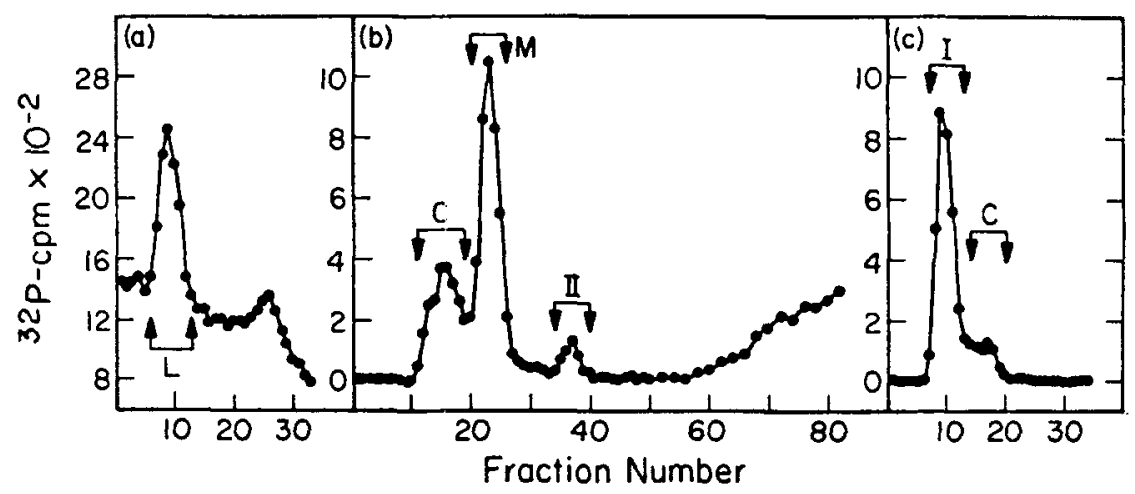

Figure 1. Purification of mtDNA from a crude mitochondrial fraction of ${ }^{32} \mathrm{P}$ labeled human (HeLa) cells by centrifugation in $\mathrm{CsCl}$, propidium diiodide gradients. (a) Buoyant density profile of the crude lysate. (b) Sedimentation profile of the material from the lower band, L, from (a). (c) Buoyant density profile of the material contained in fractions $1-32$ of (b). Electron microscopy revealed that the major constituents of the fractions pooled as $\mathrm{C}, \mathrm{M}$, and II in (b) were catenated mtDNA, monomeric closed-circular $\mathrm{mtDNA}$ and monomeric open-circular mtDNA, respectively. Similar analyses in (c) revealed closed-circular mtDNA (both catenanes and monomers) in I and replicating forms of $m$ tDNA plus catenated forms containing closed joined to open circles in $C$. The lack of detectable radioactivity in the final gradient, (c), except where expected for closed-circular, partially nicked catenated and replicating mtDNA molecules, indicates the efficiency of the purification. Each fraction represents 10 drops $(\sim 100 \mu 1)$ in (a) and (c) and 5 drops $(\sim 50 \mu 1)$ in (b). $1-\mu l$ aliquots were spotted onto Whatman GF/A filters, dried, and counted. 


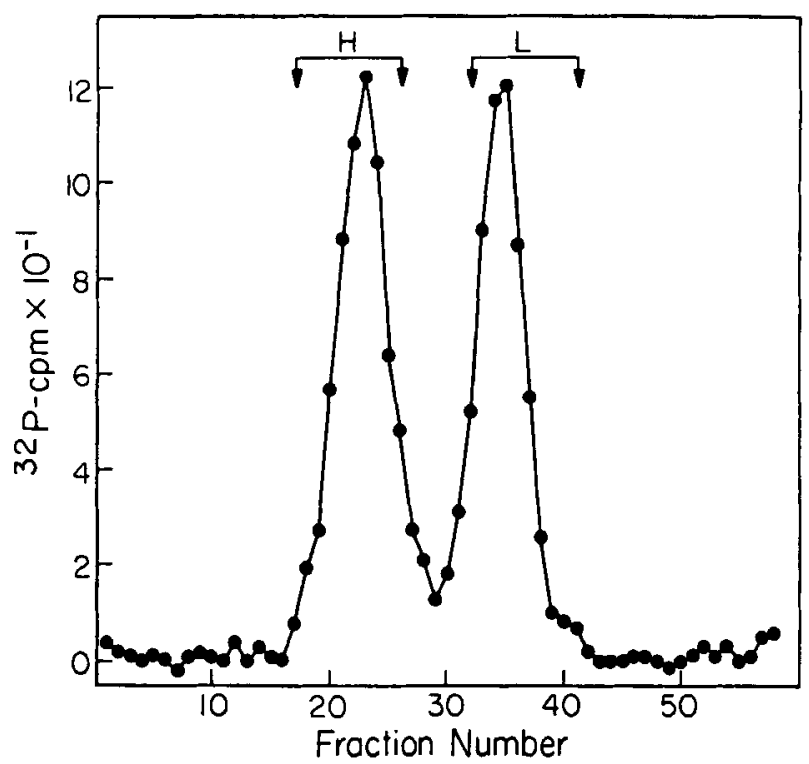

FIGURE 2. Separation of the complementary strands of ${ }^{39} \mathrm{P}$-labeled human (HeLa) mtDNA in buoyant alkaline $\mathrm{CsCl}$. The heavy $(\mathrm{H})$ and light $(\mathrm{L})$ strands were pooled as indicated. Each fraction represents 5 drops $(\sim 50 \mu \mathrm{l})$. 1- $(-\mu 1$ aliquots were taken for counting.

final sedimentation equilibrium profile (FIGURE 1c) and by the characterization with electron microscopy. ${ }^{15}$

The complementary single strands of purified mtDNA were separated in buoyant preparative alkaline $(\mathrm{pH} 12.8) \mathrm{CsCl}$ gradients and isolated by gradient fractionation as described. ${ }^{15}$ FIGURE 2 is a profile of a typical preparation, showing the fractions pooled for base compositional analysis.

\section{Base Compositional Analysis of $m t D N A$}

${ }^{32}$ P-labeled mtDNA (in $10 \mathrm{mM}$ Tris, $\mathrm{pH} 7.5,8 \mathrm{mM} \mathrm{MgCl}_{2}$ ) was digested with DNase I (Worthington Biochemical, Inc.) at $250 \mu \mathrm{g} / \mathrm{ml}$ for $75 \mathrm{~min}$ at $37^{\circ} \mathrm{C}$, then made $0.1 \mathrm{M}$ in glycine, pH 9.2 , and digested for $60 \mathrm{~min}$ at $37^{\circ} \mathrm{C}$ with $125 \mu \mathrm{g} / \mathrm{ml}$ of venom phosphodiesterase (Worthington Biochemicals, Inc.) that had been freed of detectable 5 -nucleotidase activity by the method of Sulkowski and Laskowsi. ${ }^{16}$ Samples were made $20 \mathrm{mM}$ in EDTA, the volumes were reduced to $50 \mu \mathrm{l}$ by evaporation under $\mathrm{N}_{2}$, nonradioactive marker $5^{\prime}$ deoxymononucleotides were added, and the mixture was spotted onto $7.5 \mathrm{~cm}$ wide strips of Whatman $3 \mathrm{MM}$ chromatography paper. The strips were placed in a Gilson high voltage electrophorator containing an aqueous buffer composed of $5 \%$ acetic acid, $0.5 \%$ pyridine, $10 \mathrm{mM}$ EDTA, pH 3.5, under Varsol, and electrophoresed at 3,000 volts for 90-100 min. The strips were steamed in an autoclave to remove pyridine, dried, cut into $1 \mathrm{~cm}$ slices, and the ${ }^{32} \mathrm{P}$ counted in a Beckman scintillation counter, using a toluene-based scintillation mixture. Radioactivity was always coincident with marker nucleotide fluorescence and none was detected at the migration position for free ${ }^{32} \mathbf{P}$. 


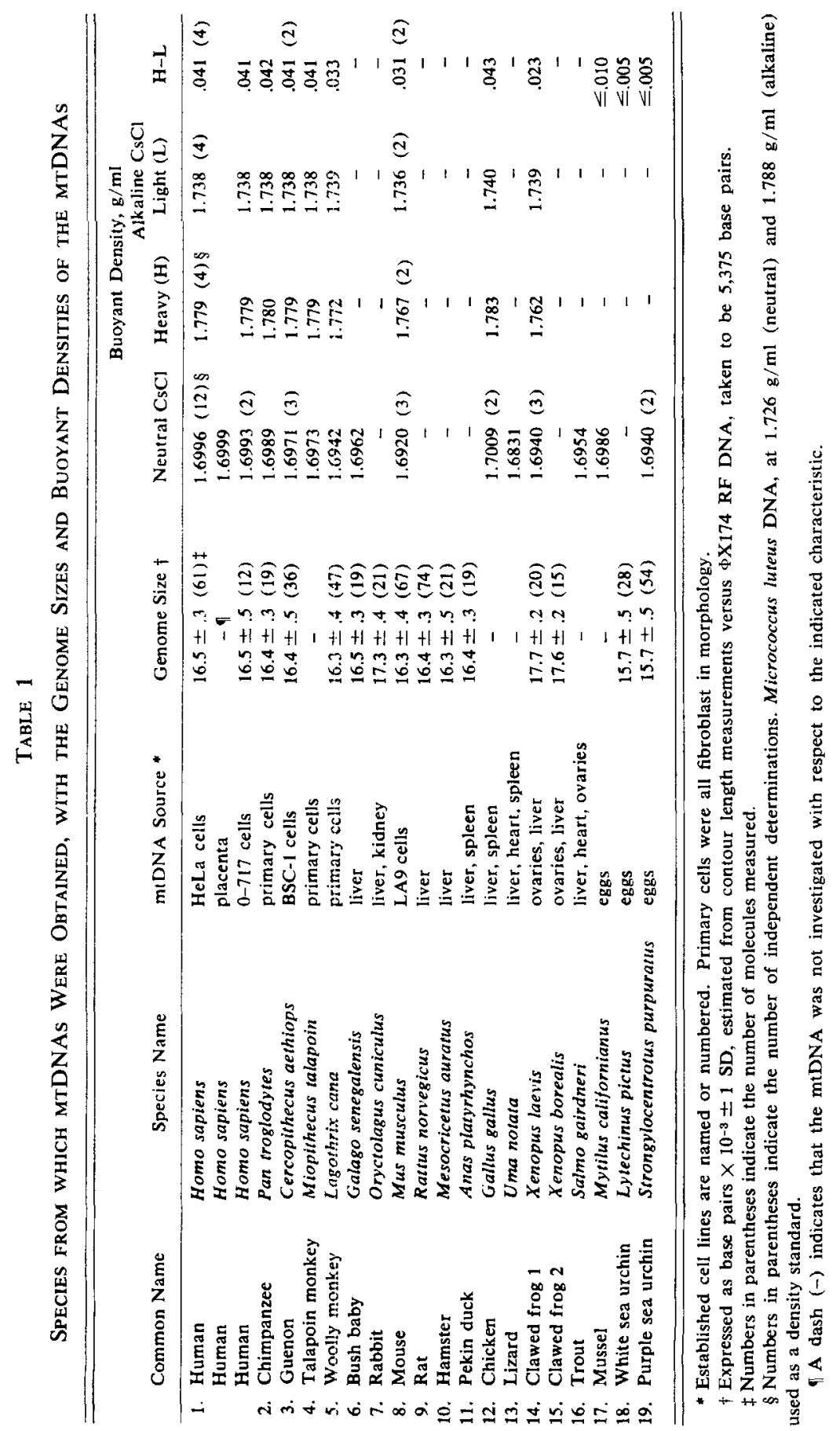




\section{Size and Buoyant Density Analysis of $m$ tDNA}

The sizes of the mtDNAs were determined by contour-length measurements from electron micrographs using bacteriophage $\emptyset \times 174$ RF DNA as an internal size standard as described. ${ }^{3,11,15}$ Buoyant density analysis in neutral and alkaline $\mathrm{CsCl}$ was performed as previously described. ${ }^{15}$ Using these conditions, $E$. coli DNA has a neutral buoyant density of $1.704 \mathrm{~g} / \mathrm{ml}$.

\section{RESULTS}

\section{Sizes of the mtDNAs}

The range in the size of the mitochondrial genome among the species examined in this study was from 15.7 to 17.7 kilobases (kb) (Table 1). Primates, rodents, and one bird species had mitochondrial genome sizes that did not differ significantly from $16.4 \pm 0.1 \mathrm{~kb}$. Among the mammals surveyed only rabbit mtDNA differed from this value, being $\sim 1 \mathrm{~kb}$ larger. The two Xenopus species surveyed had mtDNAs that were $\sim 17.6 \mathrm{~kb}$. Two distantly related sea urchin species (TABLE 1 ) have rather small mtDNAs $(\sim 15.7 \mathrm{~kb})$.

\section{Buoyant Densities of the mtDNAs}

The neutral buoyant densities of mtDNAs from 13 species were examined. The results (TABLE 1) indicate that the range of the neutral buoyant densities among the six primate mtDNAs $(1.694$ to $1.700 \mathrm{~g} / \mathrm{ml})$ is nearly the same as the range for the entire sample $(1.692$ to $1.700 \mathrm{~g} / \mathrm{ml})$. This corresponds to a $9 \%$ range in the guanine-plus-cytosine $(G+C)$ content of the mtDNAs, as determined by this method. ${ }^{17}$ The alkaline buoyant densities of mtDNAs from eight species were examined (TABLE 1). The separations of the complementary strands ranged from $23 \mathrm{mg} / \mathrm{ml}$ (Xenopus laevis) to $43 \mathrm{mg} / \mathrm{ml}$ (chicken). The separations and the respective strand densities were the same among the Old World monkeys, chimpanzee, and man (entries 1-4, TABLE 1), but both the relative strand separation and buoyant density of the heavy strand are less in woolly monkey, a New World species. In the mollusk and sea urchins examined, the mtDNAs formed a broad, flat-topped peak in buoyant alkaline $\mathrm{CsCl}$, as previously reported for one of the species ${ }^{18,19}$ with estimated stranddensity differences of less than $10 \mathrm{mg} / \mathrm{ml}$.

\section{Double- and Single-Stranded mtDNA Base Compositions}

Uniformly ${ }^{32} \mathrm{P}$-labeled mtDNAs and complementary strands from human (HeLa), guenon (BSC-1), and house mouse (LA9) cells were prepared. The DNAs were digested to 5'-mononucleotides with the enzymes DNase I and venom phosphodiesterase. After separation of the four mononucleotides by high voltage electrophoresis (FIGURE 3 ) the amount of radioactivity in each mononucleotide was counted. Results of all determinations are summarized in Figure 4 and TABLES 2 and 3. The base compositions of the primate mtDNAs were very similar, if not identical. In these DNAs, the heavy strand was more 


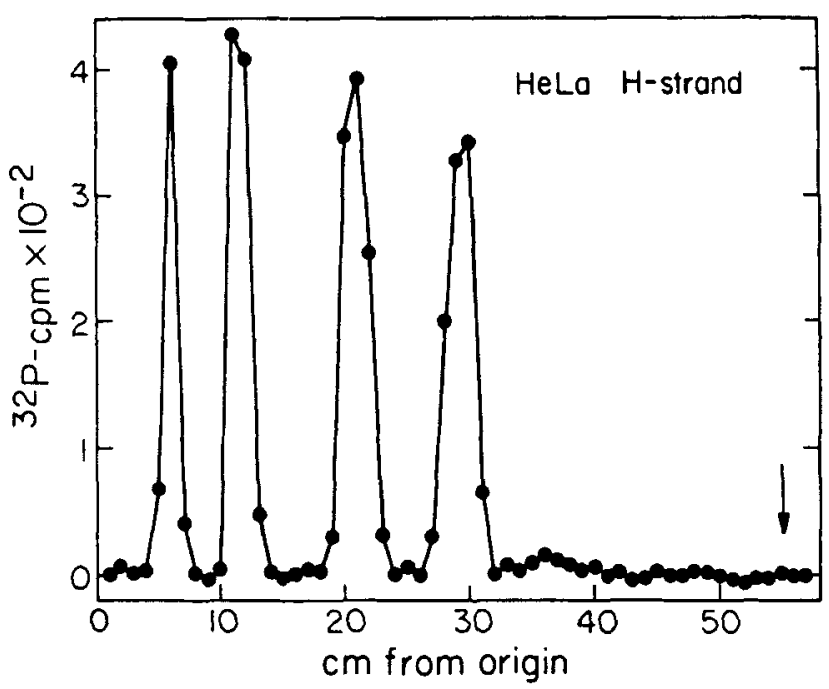

Figure 3. Separation of ${ }^{3}$ P'-labeled $5^{\prime}$-deoxymononucleotides by high-voltage electrophoresis. The nucleotides were produced by the enzymatic digestion of the heavy strand of human (HeLa) mtDNA as described in Materials aND Methods. The four peaks correspond to the 5'-monophosphates of deoxycytidine (dC), deoxyadenosine (dA), deoxyguanosine ( $\mathrm{dG}$ ), and thymidine (dT) in order of increasing mobility. The arrow indicates the peak position of ${ }^{30} \mathrm{P}_{1}$ run under identical conditions.

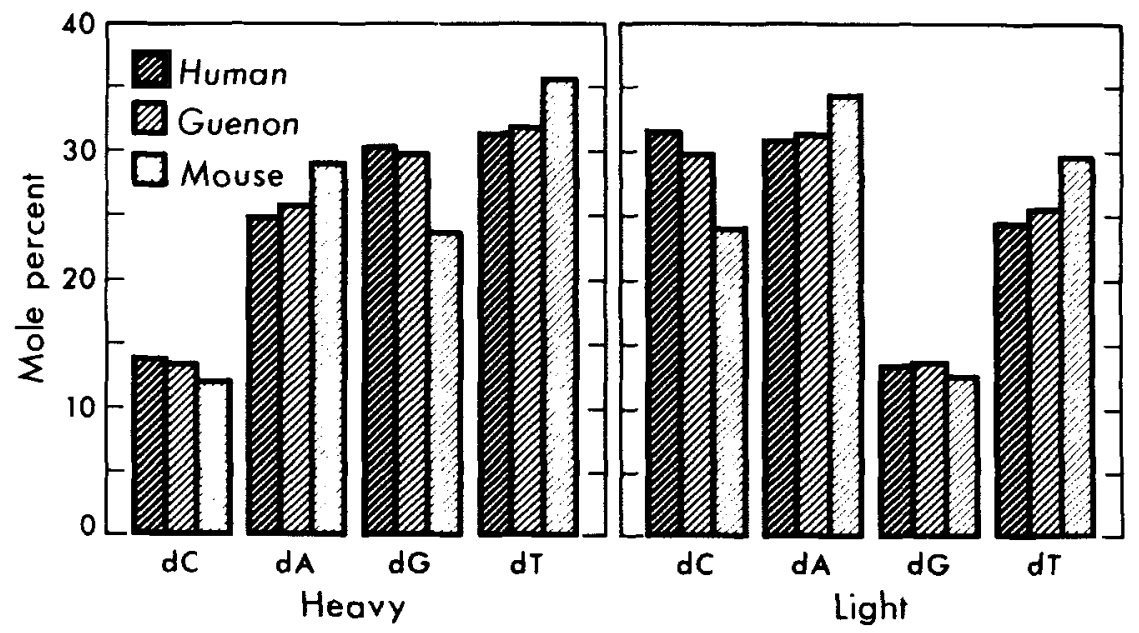

Figure 4. Base compositions of the complementary mtDNA strands of human, guenon, and house mouse. The complementary strands were prepared, digested to 5 -deoxymononucleotides and analyzed as described in Materials and Methods. The values shown are averages for multiple determinations, as detailed in TABLE 3 . The abbreviations for the four bases are given in the legend to Figure 3. 
than 2.2 times richer in guanine and more than 1.2 times richer in thymine than the light strand. Mouse mtDNA differed from these not only in the overall base composition, but also in the degree of guanine bias between the complementary strands.

\section{Discussion}

DNA undergoes two kinds of evolutionary change: structural rearrangements and base substitutions. The former includes additions (duplications), deletions, inversions, and transpositions (including translocations). Structural rearrangements occur in animal mtDNA, but such rearrangements appear to be restricted both in kind and in place of occurrence.

TABLE 2

Base Compositions of Three Native Duplex mtDNAs

\begin{tabular}{|c|c|c|c|c|c|c|c|c|c|}
\hline & \multicolumn{4}{|c|}{ Base Compositions * } & \multicolumn{5}{|c|}{ Molar Ratios $\dagger$} \\
\hline & \multirow[b]{2}{*}{$\mathrm{C}$} & \multirow[b]{2}{*}{ A } & \multirow[b]{2}{*}{ G } & \multirow[b]{2}{*}{$\mathrm{T}$} & \multirow[b]{2}{*}{ Py:Pu } & \multirow[b]{2}{*}{$\mathrm{T}: \mathrm{A}$} & \multicolumn{3}{|c|}{$(G+C)$} \\
\hline & & & & & & & $C: G$ & $\%$ & $\mathbf{N}$ \\
\hline Human & $21.9 \pm 1.4$ & $28.1 \pm 1.6$ & $22.1 \pm 1.6$ & $28.0 \pm 1.9$ & 0.99 & 1.00 & 0.99 & 44.0 & 12 \\
\hline Guenon & $21.5 \pm 0.5$ & $28.2 \pm 0.5$ & $21.7 \pm 0.5$ & $28.6 \pm 0.6$ & 1.00 & 0.99 & 0.99 & 43.2 & 5 \\
\hline Mouse & $17.8 \pm 0.5$ & $30.5 \pm 0.8$ & $19.0 \pm 0.8$ & $32.8 \pm 0.9$ & 1.03 & 0.93 & 0.92 & 36.8 & 3 \\
\hline
\end{tabular}

* Base compositions are expressed as mole $\% \pm 1 \mathrm{SD}$. C, A, G, and T refer to the 5 '-deoxymononucleotides of cytosine, adenine, guanine, and thymine, respectively.

$\dagger \mathrm{Py}: \mathrm{Pu}$ is the ratio of pyrimidines $(\mathrm{C}+\mathrm{T})$ to purines $(\mathrm{A}+\mathrm{G})$ and $\mathrm{N}$ is the number of independent determinations.

\section{Additions and Deletions Occur Predominantly in the Region of the Origin of Replication of Animal mtDNA}

The marked variation in mitochondrial genome size that exists among species (TABLE 1) is prima facie evidence for the occurrence of additions and deletions. Although apparent size homogeneity of mtDNA exists among species in some taxonomic groups, e.g., Primates, others show marked heterogeneity in size. The range of sizes reported for mtDNA among 39 species of Drosophila was 15.6 to $19.4 \mathrm{~kb},{ }^{20}$ a range greater than that for all other animal species examined. Although the available data suggest that, in general, mtDNA is homogeneous in size within species, at least one exception to this has been found. Comparison of mtDNA size among five geographically different populations of a lizard, Cnemidophorus sexlineatus indicated that individuals from one population had mtDNA that was $\sim 1200$ base pairs larger than mtDNA from individuals in the remaining populations (Brown \& Wright, unpublished data).

In a comparison of the mtDNAs of gorilla, chimpanzee, human, orangutan, and lar gibbon, gorilla mtDNA has been shown to differ in size from the remaining mtDNAs by a deletion of $\sim 100$ base pairs (Ferris, Wilson \& Brown, 


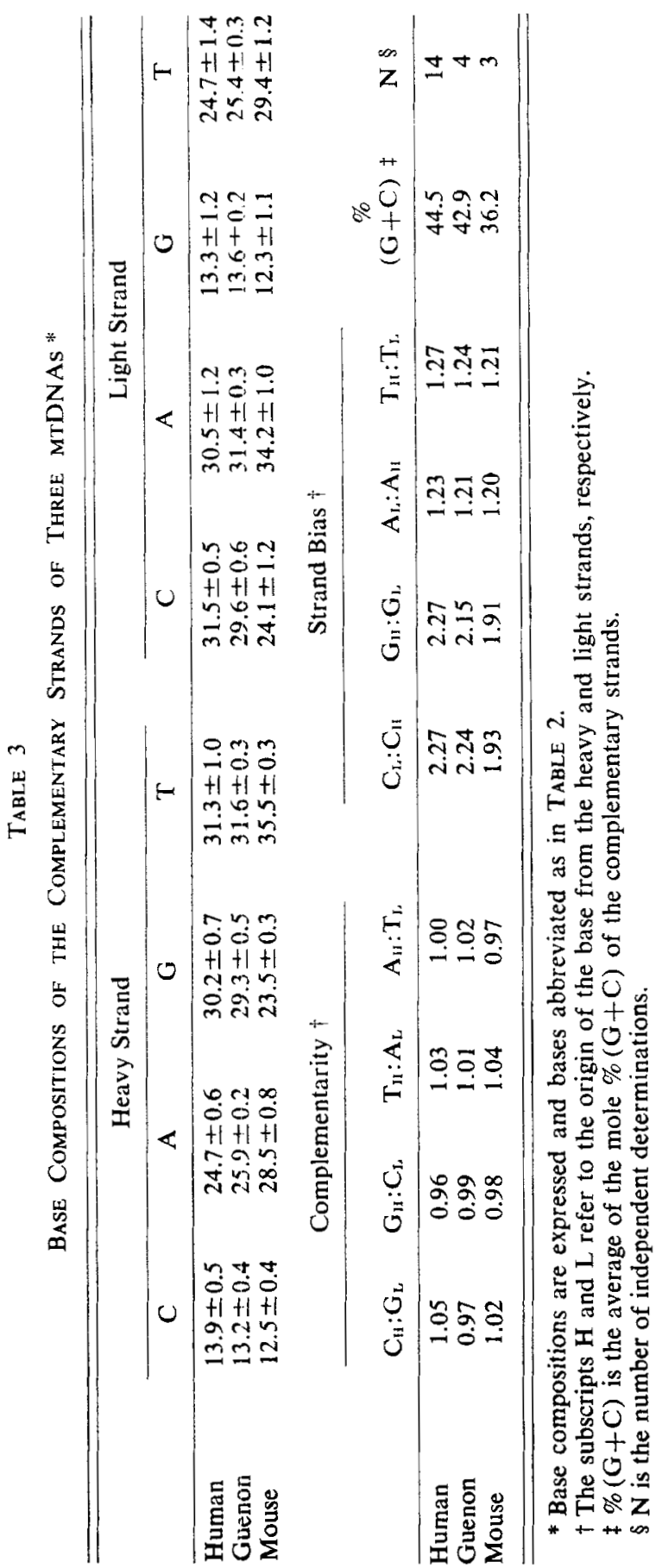


manuscript submitted). This deletion occurs in the region near the heavy strand origin of replication, or D-loop. ${ }^{22-25}$ The size difference between frog (Xenopus) and human mtDNA, $\sim 1,150$ base pairs, (TABLE 1) can be accounted for by a size difference in the regions directly adjacent to and including the D-loop. The D-loop of $X$. laevis mtDNA is $\sim 900$ base pairs longer than that of human mtDNA ${ }^{4,12,26,27}$ and the region from the proximal end of the smaller ribosomal gene to the origin of the D-loop is $\sim 300$ base pairs longer. ${ }^{4,27}$ A heteroduplex analysis of the region near the D-loop of sheep and goat mtDNA ${ }^{10}$ indicated that addition-deletion types of rearrangements were present, and possibly involved the duplication or deletion of an iterated sequence.

Contour length and restriction-endonuclease fragment size analyses indicate that most, perhaps all, of the variation observed among Drosophila species is due to differences in the size of the A+T-rich region of the mtDNA and that the remainder of the genome is not distinguishable in size among the species studied. ${ }^{20,21,28-30}$ The A+T-rich region of Drosophila mtDNA contains the origin of replication. ${ }^{21,31}$ It was suggested, based on contour-length measurements of this region, that the size differences might have arisen as duplications or deletions of an iterated unit sequence. ${ }^{20}$ However, heteroduplex studies of the A+T-rich regions indicate that there is almost no homology between species and also provide no evidence for the existence of iterated sequences within species. ${ }^{21,} 29$

Taken together, these data suggest strongly that addition-deletion events in animal mtDNA occur predominantly in the relatively small portion of the genome that includes the origin of heavy strand replication.

\section{Inversions and Transpositions May Not Occur in Animal mtDNA}

There is substantial evidence that, at least in vertebrates, the mitochondrial gene content and relative gene order is similar, perhaps identical, among different species. The relative positions of the origin of heavy strand replication and the genes coding for the small and large mitochondrial ribosomal RNAs are the same in primates, rodents, and frogs. ${ }^{2,5,6}$ The similarity in the genomic positions of the mitochondrial transfer RNA genes among representatives of these groups is also striking. ${ }^{1,4,6}$ Further evidence for the conservation of gene order in mtDNA is provided by comparisons of restriction-endonuclease cleavage maps. Although the evolutionary rate of base substitution in animal mtDNA is too high to permit long-range comparisons of cleavage sites (e.g., between primates and amphibians), such comparisons can and have been made among more closely related taxa. The first published study of mtDNA cleavage maps indicated that human, guenon, and mouse mtDNAs each contained three HindIII sites, two of which appeared to be at identical positions in the three genomes. ${ }^{11}$ Further studies have shown that HindIII sites occur at these same two positions in 10 of 11 primate species and in 3 of 5 rodent species investigated $^{3,32}$ (Ferris, Wilson \& Brown, manuscript submitted; S. Ferris and M. George, Jr., unpublished data). Other highly conserved sites have also been identified. These sites occur infrequently and are distributed at positions throughout the genome, outside of as well as within the mitochondrial ribosomal RNA genes. These data suggest that the order of sequences in the mitochondrial genome is conserved and that transpositions and inversions have not occurred in these taxa. 
Finally, a hybridization study ${ }^{33}$ in which each of the labeled, separated strands of human mtDNA annealed exclusively with the corresponding complementary strand of frog (Xenopus laevis) mtDNA indicates that the hybridizable sequence information contained in each of the complementary strands has remained in that strand since the divergence of the human and frog lines from a common ancestor, an event that occurred $\sim 3.5 \times 10^{8}$ years ago. ${ }^{34}$

Studies of mtDNA transcription in both house mouse ${ }^{5}$ and Xenopus ${ }^{4}, 27$ indicate that intervening sequences are probably not present, at least not in the genes for ribosomal or messenger RNAs. No intervening sequences occur in the COII gene of human mtDNA or in the transfer RNA genes flanking it. ${ }^{35}$ These studies, and others dealing with the location of transfer RNA genes, ${ }^{1,4,6}$ also indicate that intergenic spacer regions are extremely small or absent. This has been directly confirmed by partial sequence data for human ${ }^{35}$ and house mouse (D. Clayton, personal communication) mtDNAs. This contiguous arrangement of the genes may be the reason for the lack of occurrence of structural rearrangements outside the region of the heavy strand origin of replication in animal mtDNA, since any rearrangement outside this region would almost certainly inactivate one or more genes.

\section{Sequence Evolution in Animal mtDNA Is Rapid}

Despite the apparent conservation of gene content and order in animal mtDNA, the rate of base substitution appears to be rapid. The substitution rate, estimated to be 0.5 to $1 \%$ per genome per $10^{6}$ years, is 5 to 10 -fold faster than the rate for single-copy nuclear DNA, based on a comparison among mammals. ${ }^{3}$ A similar rate estimate is obtained using data from a comparison of the mtDNAs from two amphibian species (Xenopus laevis and $X$. borealis) ${ }^{8}$ that diverged $\sim 10 \times 10^{6}$ years ago. ${ }^{36}$ Thermal dissociation of mtDNAs hybridized in vitro ${ }^{3, s, 33}$ and comparisons of cleavage maps ${ }^{3}$ (Ferris, Wilson \& Brown, submitted) indicate that all portions of the mitochondrial genome accumulate base substitutions relatively rapidly, at least among vertebrate species. No evidence for clustering of changed or conserved sequences is provided by either method of analysis, although both indicate that some rate heterogeneity exists for different regions of the mtDNA. The available evidence indicates that the genes for mitochondrial ribosomal RNA ${ }^{8,30}$ (Ferris, Wilson \& Brown, submitted) and transfer RNA ${ }^{37}$ are relatively more conserved than other mtDNA sequences, but that even these genes evolve much more rapidly than their nuclear counterparts.

Among species of invertebrates, only the mtDNA of Drosophila has been well studied. In this genus, the $\mathrm{A}+\mathrm{T}$-rich region has been shown by heteroduplex analysis to change much more rapidly than the remainder of the mtDNA. ${ }^{21,29}$ The rate of base substitution for Drosophila mtDNA is unknown. However, a comparison of cleavage maps for four restriction endonucleases in mtDNAs of three Drosophila species has been made. ${ }^{30}$ These data were compared with comparative mobility data from starch-gel electrophoresis of proteins, and it was concluded that the rate of base substitution in Drosophila mtDNA (exclusive of the A+T-rich region) was the same as the rate for nuclear genes. ${ }^{30}$ This is a surprising result, since it is very different than the result obtained from comparisons of vertebrate DNAs. It is especially surprising, however, because the coding regions of nuclear genes (as represented by 
mature, cytoplasmic messenger RNAs) have been shown to accumulate base substitutions more slowly than does single-copy nuclear DNA, ${ }^{38}$ which contains both coding and noncoding regions (e.g., intervening and flanking "spacer" sequences). While it is possible that the substitution rate varies among different taxonomic groups, to draw such a conclusion now would be premature. The Drosophila study included relatively few cleavage sites for comparison; this can lead to a large error. ${ }^{39}$ Also, the comparison was indirect, with nuclear proteins, rather than direct, with nuclear DNA. This result is, nevertheless, highly interesting and a more rigorous comparison ought to be undertaken.

\section{Changes in the Distribution of Bases between Strands}

All animal mtDNAs so far examined show an unequal distribution of guanine plus thymine residues between the complementary strands. This has been demonstrated both directly, by base compositional analysis (TABLE 3) and indirectly, by equilibrium sedimentation in alkaline $\mathrm{CsCl}$ gradients (TABLE 1). The difference in the buoyant densities of the complementary strands in alkaline $\mathrm{CsCl}$ can vary among animal species from $\leq 5$ to $43 \mathrm{mg} / \mathrm{ml}$ (TABLE 1). The invertebrate species show much smaller interstrand differences than do the vertebrate species. Among vertebrates, the "warm blooded" species (birds and mammals) exhibit greater interstrand differences than does the one "cold-blooded" species examined (Xenopus). Although the amount of data available is limited, one may speculate that there is an evolutionary trend toward an increasing strand bias for guanine plus thymine content in going from invertebrates to vertebrates and, within the vertebrates, in going from less metabolically active, "cold-blooded" forms to more metabolically active, "warm blooded" forms.

\section{How Are Rapid Changes in mtDNA Generated?}

It seems unlikely that the rates of mitochondrial and nuclear mutations caused by external physical events (e.g., radiation) or by externally applied chemical mutagens would differ (although one could argue that mtDNA, being shielded by an additional membrane barrier, might be less exposed to chemical mutagens). It has been proposed, however, that chemical events within the mitochondrion result in the production of mutagenic compounds, such as free radicals. ${ }^{40}$ If this proposal is correct, these would increase the rate of substitutions in mtDNA without affecting nuclear DNA, since the mutagenic compounds generated would be confined to the mitochondrion.

DNA base substitutions can arise as a result of errors generated during replication. There is evidence that in vertebrates the occurrence of replicationgenerated errors is higher in mtDNA than in nuclear DNA. The incorporation of ribonucleotides into replicating mtDNA and the presence of these in mature (i.e., nonreplicating) mtDNA have been well documented.41 Although the frequency of ribonucleotide incorporation may be greatest near the respective origins of replication of the two strands, ${ }^{42,43}$ ribonucleotides also occur more or less randomly throughout the remainder of the genome. ${ }^{33,44}$ The presence of ribonucleotides in mature mtDNA suggests strongly that the enzymatic replication complex for mtDNA, unlike its nuclear counterpart, is either inefficient or 
lacking in the ability to edit newly-replicated DNA. Unlike vertebrate mtDNA, mature Drosophila mtDNA is not susceptible to strand scission at high $\mathrm{pH}$, and therefore does not appear to contain ribonucleotides ( $J$. Battey, personal communication).

The turnover rate of mtDNA is higher than that of nuclear DNA in the somatic cells of vertebrates. ${ }^{45}$ If this is also true for germ-line cells it would contribute to a higher substitution rate, since mtDNA would undergo more rounds of replication than nuclear DNA, thus providing more opportunities for the occurrence of replication-associated substitutions.

The apparent lack of post-replicative repair mechanisms in vertebrate mitochondria may also contribute to the elevated rate of base substitution in mtDNA. No mechanism for the excision and repair of ultraviolet radiationinduced thymine dimers is demonstrable. ${ }^{16}$ Studies of the ability of thymine kinase-deficient cells to recover from photoinduced lesions in bromouracilsubstituted mtDNA indicate indirectly that mechanisms for the efficient repair of these lesions are lacking. ${ }^{4 i}$ The persistence of ribonucleotide bases in mtDNA also indicates the lack of efficient post-replicative repair mechanisms in vertebrate mitochondria.

In cultured mammalian cells, both replicative and post-replicative repair syntheses of DNA have been shown to depend on the presence of the enzyme $\alpha$-DNA polymerase. ${ }^{+5}$ No DNA repair was observed in its absence, even though $\beta$ - and $\gamma$-polymerase were present. ${ }^{4 s}$ In vertebrates, only the $\gamma$-polymerase has been isolated from mitochondria and this enzyme seems to be the only polymerase involved in the replication of mtDNA. ${ }^{+9}$ The lack of both replicative and post-replicative repair of vertebrate mtDNA may thus be due to the lack of an $\alpha$-polymerase in the mitochondria.

Three forms of DNA polymerase have been obtained from Drosophila. ${ }^{50,51}$ Unlike the separately coded polymerases in vertebrates, in Drosophila the three activities appear to result from the specific proteolysis of the product of a single gene product. ${ }^{51,52}$ It is not known which form of polymerase is associated with mitochondria. If the rate of nucleotide substitution in Drosophila mtDNA differs from that in vertebrate mtDNA, characterization of the Drosophila polymerase and comparison of it with vertebrate $\gamma$-polymerase can provide not only a mechanistic interpretation for any rate difference, but also a quantitative understanding of the role that DNA polymerases play in the generation of mutations.

\section{The Rate of Evolution of Vertebrate mtDNA May Be Mutationally Driven}

The rate of evolution is the product of the separate rates for mutation and fixation. In comparisons of nuclear genes, a rate difference is usually attributed to a difference in the rate of fixation, due to functional constraints imposed by selection on the gene products. Mitochondrial gene products function as components in complex, multisubunit structures (e.g., ribosomes, cytochrome oxidase complex, etc.), most of the components of which are products of nuclear genes. It is thus difficult to accept an argument for lower functional constraints on mitochondrial gene products as a valid explanation for the high rate of mtDNA evolution. It seems at least as likely that the rapid evolution of vertebrate mtDNA is due exclusively to its higher mutation rate and that the 
rate of fixation is the same as that for an analogous set of nuclear DNA sequences.

\section{ACKNOWLEDGMENTS}

I am deeply grateful to the late Jerome Vinograd, Divisions of Biology and Chemistry, California Institute of Technology, Pasadena; to Howard M. Goodman, Department of Biochemistry and Biophysics, University of California, San Francisco; and to Allan C. Wilson, Department of Biochemistry, University of California, Berkeley, for their interest, support and encouragement of my research and for the use of their facilities; to Jim Battey, David Clayton, Steve Ferris, and Matt George for sharing their unpublished results; to Allan Wilson, David Clayton, C. Allen Smith, Harold Varmus, John Wright, and Susan Brown, and to the members of the Vinograd, Goodman, and Wilson labs for many helpful and enlightening discussions; and to Lori Erdley for assistance in the preparation of this paper.

\section{REFERENCES}

1. Dawid, I. B., C. K. Klukas, S. Ohi, J. L. Ramirez \& W. B. Upholt. 1976. In The Genetic Function of Mitochondrial DNA. C. Saccone \& A. M. Kroon, Eds. Elsevier, Amsterdam.

2. Ramirez, J. L. \& I. B. Dawid. 1978. J. Mol. Biol. 119:133-146.

3. Brown, W. M., M. George, Jr. \& A. C. Wilson, 1979. Proc. Natl. Acad. Sci. USA 76:1967-1971.

4. Rastl, E. \& I. B. Dawid. 1979. Cell 18:501-510.

5. Battey, J. \& D. A. Clayton. 1978. Cell 14:143-156.

6. Attardi, G., M. Albring, F. Amalric, R. Gelfand, J. Griffith, D. Lynch, C. Merkel, W. Murphy \& D. OJala. 1976. In Genetics and Biogenesis of Chloroplasts and Mitochondria. T. Bucher, W. Neupert, W. Sebald \& S. Werner, Eds. Elsevier, Amsterdam.

7. Borst, P. 1977. Trends in Biochem. Sci. 2:31-34.

8. DawID, I. B. 1972. Devel. Biol. 29:139-151.

9. Jakovcic, S., J. Casey \& M. Rabinowitz. 1975. Biochemistry 14:2037-2042.

10. Upholt, W. B. \& I. B. DAwID. 1977. Cell 11:571-583.

11. Brown, W. M. \& J. Vinograd. 1974. Proc. Natl. Acad. Sci. USA 71:4617-4621.

12. Brown, W. M., J. Shine \& H. M. Goodman. 1978. Proc. Natl. Acad. Sci. USA 75:735-739.

13. Brown, W. M. \& H. M. Goodman. 1979. In Extrachromosomal DNA. D. Cummings, P. Borst, I. Dawid, S. Weissman \& C. F. Fox, Eds. Vol. 15:485-500. Academic Press, Inc. New York, N.Y.

14. Brown, W. M. \& J. W. WRIGHT. 1979. Science 203:1247-1249.

15. Brown, W. M., R. M. Watson, J. Vinograd, K. M. Tait, H. W. Boyer \& H. M. Goodman. 1976. Cell 7:517-530.

16. Sulkowski, E. \& M. Laskowski, SR. 1971. Biochim. Biophys. Acta 240:443447.

17. Schildkraut, C. L., J. Marmur \& P. Doty. 1962. J. Mol. Biol. 4:430-443.

18. Piko, L., A. Tyler \& J. Vinograd. 1967. Biol. Bull. 132:68-90.

19. Piko, L., D. G. Blair, A. Tyler \& J. Vinograd. 1968. Proc. Natl. Acad. Sci. USA 59:838-845.

20. Fauron, C. M-R. \& D. R. Wolstenholme. 1976. Proc. Natl. Acad. Sci. USA 73:3623-3627. 
21. Wolstenholme, D. R., J. M. Goddard and C. M-R. Fauron. 1979. In Extrachromosomal DNA. D. Cummings, P. Borst, I. Dawid, S. Weissman \& C. F. Fox, Eds. Vol. 15:409-425. Academic Press, Inc. New York, N.Y.

22. Kasamatsu, H., D. L. Robberson \& J. Vinograd. 1971. Proc. Natl. Acad. Sci. USA 68:2252-2257.

23. Robberson, D. L., H. Kasamatsu \& J. Vinograd. 1972. Proc. Natl. Acad. Sci. USA 69:737-741.

24. Kasamatsu, H. \& J. Vinograd. 1973. Nature New Biol. 241:103-105.

25. Robberson, D. L. \& D. A. Clayton. 1972. Proc. Natl. Acad. Sci. USA 69: 3810-3814.

26. Gillum, A. M. \& D. A. Clayton. 1978. Proc. Natl. Acad. Sci. USA 75:677681.

27. Rastl, E. \& I. B. DawiD. 1979. In Extrachromosomal DNA. D. Cummings, P. Borst, I. Dawid, S. Weissman \& C. F. Fox, Eds. Vol. 15:395-407. Academic Press, Inc. New York, N.Y.

28. Goddard, J. M. \& D. R. Wolstenholme. 1979. J. Cell Biol. 83(2): Abstr. MC1936.

29. Fauron, C. M-R. \& D. R. Wolstenholme. 1979. J. Cell Biol. 83(2): Abstr. MC1937.

30. Shah, D. M. \& C. H. Langley. 1979. Nature 281:696-699.

31. Goddard, J. M. \& D. R. Wolstenholme. 1978. Proc. Natl. Acad. Sci. USA 75: 3886-3890.

32. Parker, R. C. \& R. M. Watson. 1977. Nucleic Acids Res. 4(5):1291-1299.

33. Brown, W. M. 1976. Ph.D. Thesis. California Institute of Technology. Pasadena, Calif.

34. Romer, A. S. 1966. Vertebrate Paleontology. University of Chicago Press. Chicago, Ill.

35. Barrell, B. G., A. T. Bankier \& J. Drouin. 1979. Nature 282:189-194.

36. Bisbee, C. A., M. A. Baker, A. C. Wilson, I. Hadji-Azimi \& M. Fischberg. 1977. Science 195:785-787.

37. Jakovcic, S., J. Casey \& M. Rabinowitz. 1975. Biochemistry 14:2043-2050.

38. Rosbash, M., M. S. Campo \&. K. S. Gummerson. 1975. Nature 258:682-686.

39. NeI, M. \& W-H. Lr. 1979. Proc. Natl. Acad. Sci. USA 76:5269-5273.

40. Borst, P. \& A. M. Kroon. 1969. Int. Rev. Cytol. 26:107-190.

41. Grossman, L. I., R. M. Watson \& J. Vinograd. 1973. Proc. Natl. Acad. Sci. USA 70:3339-3343.

42. Martens, P. A. \& D. A. Clayton. 1979. J. Mol. Biol. 135. (In press.)

43. Gillum, A. M. \& D. A. Clayton. 1979. J. Mol. Biol. 135. (In press.)

44. Flory, P. J., JR. 1974. Ph.I. Thesis. California Institute of Technology. Pasadena, Calif.

45. Rabinowitz, M. \& H. Swift. 1970. Physiol. Rev. 50:376-427.

46. Clayton, D. A., J. N. Doda \& E. C. Friedberg. 1974. Proc. Natl. Acad. Sci. USA 71:2777-2781.

47. Lansman, R. A. \& D. A. Clayton. 1975. J. Mol. Biol. 99:761-776.

48. Cinrrocchi, G., J. G. Jose \& S. Linn. 1979. Nucleic Acids Res. 7(5):12051219.

49. Weissback, A. 1977. Annu. Rev. Biochem. 46:25-47. E. Snell, P. Boyer, A. Meister and C. Richardson, Eds. Annual Reviews, Inc. Palo Alto, CA.

50. Brakel, C. L. \& A. B. Blumenthal. 1977. Biochemistry 16:3137-3143.

51. Brakel, C. L. \& A. B. Blumenthal. 1978. Eur. J. Biochem. 88:351-362.

52. Brakel, C. L. \& A. B. Blumenthal. 1978. Nucleic Acids Res. 7(5):2565-2575. 


\section{Discussion of the PaPeR}

DR. D. RuIz: Continuing on your speculation about the higher rate of fixation of mutations in the mitochondria, it is intriguing to me to postulate that there could be a much higher rate of fixation of mutations based on the fact that selective pressure against an unfavorable mutation would not be nearly so high as against a mutation in the nuclear DNA which only exists in one copy.

DR. W. M. BRown: I forgot to mention that there are several hundred copies of mitochondrial DNA in the several hundred mitochondria per cell.

DR. RuIz: The same kind of theory that has been applied to greater rates of evolution in duplicated genes in the nuclear DNA could be applied to this sort of situation.

DR. Brown: I think that in nuclear DNA the situation is that duplicated genes evolve much more slowly. That's certainly the case with ribosomal genes. In fact, the mechanism that has been postulated is unequal crossing over for keeping things the same. You see these, for instance, in duplicate genes like duplicate alphaglobin genes.

DR. R. SAGER: I wanted to return to this question of heterogeneity because I think it is really a fascinating and very important question. How, in the absence of good repair system, is the amount of homogeneity retained that is retained? We've been looking at mouse cell lines with a somewhat more sensitive method. What we found with the cell lines that we've been working with is that there is some micro-heterogeneity. The reason I am bringing this up now is that there is a very perplexing genetic question. We've been selecting mutations to antibiotic resistance which we think are mitochondrial such as chloramphenicol resistance, oligomycin, and a few others; and they can be selected without mutagenizing the cells. This has suggested to us that there is some genetic heterogeneity there that we are selecting. Chloramphenicol resistance is very dominant in the sense that you don't need many copies to confer resistance to the cell. Oligo is different. You need a lot of copies. So we can watch segregation and where did it come from? I think it must be coming from some heterogeneity that hasn't been detected.

Dr. Brown: How many cell generations are there between the time you start the selection and the time you actually see colonies?

DR. SAGER: I can't give you an easy answer to that.

DR. BRown: I know that the mutations to antibiotic resistance occur at a very low frequency so it is possible that they are occurring during the selection procedure itself. You know, that's an alternative.

I am really starting to work on the problem within individual heterogeneity in a serious way and $I$ hope to have some data soon that will sort of indicate what's going on.

DR. M. V. Simpson: I thought in this connection I would remind everyone of a fascinating experiment done many years ago by David Luck in which he introduced into a healthy Neurospora cell a sick mitochondrion. This mitochondrion was not a pokey, but, it was a pokey-like mutant and in three generations the Neurospora caught the disease. All the mitochondria in the Neurospora had mutated. It was a rather fascinating experiment.

Dr. Brown: We really need to know something about the transmission genetics of animal mitochondrial DNA.

Dr. K. S. Chiang (University of Chicago, Chicago, Ill.): I am also 
interested in the micro-heterogeneity. I wonder whether you would like to comment on whether micro-heterogeneity is necessarily coming from the mechanism you suggested. I think the question of whether or not the organelle unit has a repair system has to be scrutinized more carefully. Whether mitochondria have a similar trend with lower UV doses, I think is an open question. Micro-heterogeneity does exist. Perhaps the mechanism for explaining such existence should be broadened rather than saying only that repair is absent.

DR. Brown: You still have to be able to explain the retention of ribonucleotides in mature mitochondrial DNA. I think that's pretty strong evidence that the repair system is either not there at all or very inefficient. 